\title{
A MICROSTRUCTURAL MODEL BY SPACE TESSELLATION FOR A SINTERED CERAMIC: CERINE
}

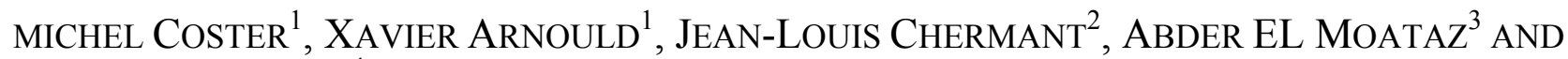 \\ THIERRY CHARTIER ${ }^{4}$ \\ ${ }^{1}$ Université de Caen and SIFCOM, UMR CNRS 6176, 6 Bd Maréchal Juin, 14032 Caen Cedex, France; \\ ${ }^{2}$ LARMAUR, FRE CNRS 2717, Bât 10B, Université de Rennes1, Campus de Baulieu, 35042 Rennes Cedex, \\ France; ${ }^{3}$ GREYC, UMR CNRS 6072, ENSI Caen, 6 Bd Maréchal Juin, 14050 Caen Cedex, France; ${ }^{4}$ SPCTS, \\ UMR CNRS 6638, ENSCI, 47-73 Avenue A. Thomas, 87065 Limoges Cedex, France \\ e-mail: Michelcoster@aol.com \\ (Accepted May 24, 2005)
}

\begin{abstract}
This paper deals with the use of probabilistic models such as Voronoi tessellation and the Johnson-Mehl model to simulate microstructures of monophased materials. Sintered cerine was chosen to test the various models. The values of morphological parameters measured, both on such models and real microstructures are in very good agreement. An extension for porous materials is also presented. This work shows that the probabilistic models are very well suited for the simulation and description of ceramic and granular microstructures.
\end{abstract}

Keywords: cerine, Johnson-Mehl model, mathematical morphology, microstructural and probabilistic models, sintered ceramics, Voronoi tessellation.

\section{INTRODUCTION}

Two strategies exist to describe the microstructure of a material: either to undertake an exhaustive morphological analysis or to use a model to describe the microstructure. The complete morphological analysis requires generally a large number of functions and parameters.

There are several methods of modelling a microstructure. The first method and the most intuitive is the physical approach: it consists of considering that a microstructure can be modelled using simple geometrical objects (spheres, cylinders ...). These objects are assembled initially in a random manner and can be positioned in order to simulate the formation of a material, following physical laws, such as densification (Stroeven and Stroeven, 1996; Stroeven, 1999; Dinger, 2000).

A similar approach has been used to describe the setting of cement during hydration by adjusting the model with three morphological parameters: the volume fraction, $\mathrm{V}_{\mathrm{V}}$, the specific surface area per unit volume, $\mathrm{S}_{\mathrm{V}}$, and the grain size distribution in $2 \mathrm{D}$ (Garboczi and Bentz, 1993; Bentz, 1997). Consequently, the resultant structure is modified by a virtual hydration. The inconvenience of such an approach is the necessity to conceive specific simulations difficult to test by image analysis (the tests have no direct relation to significant parameters and are thus not sufficiently discriminating to validate the model).

The second approach, frequently employed, is to start with a tessellation in space. This can be obtained by labelling the voxels related to a crystal orientation, called spin. The grains are formed by all the voxels with the same adjacent spin. This approach enables a test of the methods of construction by using the 3D characteristics deduced from 2D size distribution by image analysis (Mehnert et al., 1998). Simulations by the Monte Carlo method have also served to study solid phase sintering (Matsubara et al., 1999) and also grain growth in a monophased system (Xiaoyan and Liu, 1998). The space tessellation most often used is that of Voronoi. Here it serves from the beginning to carry out virtual sintering with a liquid phase (Tikare et al., 1998a, b). The Voronoi tessellation likewise serves to characterize and analyse the mechanical behaviour of heterogeneous microstructures (Ghosh et al., 1997a, b). This second method resembles that which we propose to use: probabilistic methods such as those of Poisson tessellations (mosaic) (Xi et al., 1996). 
The probabilistic models are well suited for the simulation of many materials (Jeulin, 1991), or even of their surface state (Jeulin and Laurenge, 1996; Aubert and Jeulin, 2000). The comparison between real microstructures and those given by such probabilistic models permits a correct and complete description of the microstructures only from few parameters (Jeulin, 1991; Coster and Chermant, 2002). These probabilistic models include most part of tessellations. As for example, only one parameter is requested for Voronoi tessellation: the point density; for the classical Johnson Mehl tessellation, two parameters are required, the germination rate and the growth rate.

In this paper, we shall present this last approach, using as an example, a sintered ceramic: cerine, $\mathrm{CeO}_{2}$. Sintering $\mathrm{CeO}_{2}$ has already been investigated by Arnould (Arnould et al., 2001; Arnould, 2002; Arnould et al., 2002; Coster et al. 2005), using segmented images, as described in these references.

The aim is to present to the ceramic and material science community how one can use probabilistic models to describe the morphology of granular materials in terms of the statistical structure, i.e., without taking into account the "defects" of materials such as anomalous grain size, cracks etc., and to access 3D stereological parameters. At present the ceramic industry pays a lot of attention to the morphological parameters of the initial powders and to the process to avoid such defects.

Several theoretical publications have considered modelling of the morphology of materials from random closed sets (RACS) (Matheron, 1975; Stoyan et al., 1995; Jeulin, 1997a; Stoyan, 1998). Matheron (1975) who devised the Boolean approach was the leader in this field. His work has led to numerous and important developments, substantially described by Serra (1982; 1988) and, more specially, by Jeulin (1997b).

\section{PROBABILISTIC MODELS}

Firstly, we shall inform the non-familiar readers of the main probabilistic models.

\section{POINT PROCESS}

The main probabilistic models are constructed by a random point process: the Poisson point process.

The probability that $\mathrm{n}$ points of the Poisson process of density $\theta$ belong to a set $\mathrm{Z}$ is given by:

$$
P_{n}(Z)=\frac{\left(\theta \operatorname{Mes}(Z)^{n}\right)}{n !} \exp (-\theta \operatorname{Mes}(Z))
$$

where "Mes" means measure of set Z: (volume in $\mathbf{R}^{3}$, area in $\mathbf{R}^{2}$, length in $\mathbf{R}^{1}$ ).

The advantage of the Poisson point process is that it is possible to process point densities totally independently from one frame of measurement of reference to another. It enables derived point processes to be constructed, such as a set of points with repulsion (hardcore model) or, with attraction (cluster model) (Stoyan et al., 1995).

\section{THE DIFFERENT CLASSES OF PROBABILISTIC MODELS}

The probabilistic set of models can be subdivided into two main categories: the "monophased" models which correspond only to a random tessellation of space and "polyphased" models. Moreover, modelling can be achieved "instantaneously" or "sequentially".

Table 1 summarises the main models. During sintering, the system evolves and can be considered as polyphased (solid phase and pores), whereas towards the end of sintering the material becomes monophased. In order to choose the correct model, the following approach has been proposed:

(i) to construct a model for a monophased material,

(ii) to implant a network of pores to describe the final stages of sintering.

This implies that it is not necessary to construct a model to describe the first stages of sintering. Of course, it is not necessary to have a single model to describe all stages of sintering. Thus the initial stages will be incorporated in the group of space tessellations irrespective of whether with instantaneous or sequential processes.

The Poisson tessellation, developed by Matheron (1975) and Miles (1971) and used by Xi et al. (1996), has not been considered here, as it does not correspond to granular structures of monophased ceramics (alignments of tessellations).

The monocoloured dead leaves model (Jeulin, 1997b) can also be ignored as it leads to grains totally included within others, which does not correspond to the morphology of ceramics. Thus, only two possible basic models remain, which are well known:

- the Voronoi model and,

- the Johnson-Mehl model. 
Table 1. The main groups of models.

\begin{tabular}{|c|c|c|}
\hline & Monophased models & Polyphased models \\
\hline \multirow{6}{*}{ 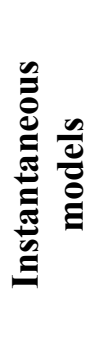 } & Space tessellation & Space tessellation \\
\hline & Voronoi model & Coloured tessellations \\
\hline & Poisson mosaic & (Voronoi, Poisson) \\
\hline & & Granular models \\
\hline & & Boolean model \\
\hline & & Stienen model \\
\hline \multirow{4}{*}{ 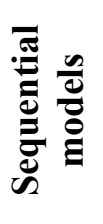 } & Space tessellation & \\
\hline & Johnson-Mehl model & \\
\hline & Granular models & Granular models \\
\hline & Monocoloured dead leaves model & Coloured dead leaves model \\
\hline
\end{tabular}

\section{HOW TO TEST A TESSELLATION MODEL?}

Before discussing the chosen models, it is necessary to show how we can validate the choice of a tessellation model. In fact, a visual comparison only is insufficient: a comparison must be quantified. The strategy must depend on a certain number of properties of the models. In this work, the most important properties will be the infinite divisibility and the calculability.

Voronoi and Johnson-Mehl models are two tessellations, which can be modified if necessary. To choose the best model and to fit its parameters, one must compare quantitatively the morphology of the model to that of the material microstructure. Then the model could be validated if a sufficient number of parameters or of morphological functions lead to the same results for the material and the model. To realize this comparison and to choose the best strategy, a certain number of properties of the tested models must be investigated. So, if we can define the relationship of a morphological function measurable in 2D from the 3D parameters of the model, it will be not necessary to carry out a 3D simulation of the model and to make measurements on $2 \mathrm{D}$ section and to compare these measurements to that of the ceramographic sections (calculability of the model). That is the case of some models such as the Boolean models or the Poisson tessellations, which give 2D sections of same nature (infinite divisibility of the model). The morphological functions are the Choquet capacities or their derivatives, which can be estimated from measurements on eroded sets. Unfortunately the Voronoi and Johnson-Mehl tessellations do not possess these properties. So, one must carry out 3D simulations and extract 2D sections to undertake measurements and to compare them with that of the material sections. The estimation of one morphological function only is generally not sufficient to validate a model. In fact for the Voronoi and Johnson-Mehl tessellations, the $\mathrm{P}(\ell)$ function or its opposite 1- $\mathrm{P}(\ell)$ are only known.

A random set possesses the properties of calculability if we can define for certain compacts (also called structuring elements in mathematical morphology), K, some explicit expressions of the Choquet capacity (Choquet, 1953). The Choquet capacity for a compact (structuring element) $\mathrm{K}$ is the probability that a compact hits the random set $\mathrm{X}$ :

$$
\mathrm{T}(\mathrm{K})=\operatorname{Pr}(\mathrm{X} \cap \mathrm{K} \neq \varnothing) .
$$

That Choquet capacity is easily estimated from simple compacts used in mathematical morphology (Serra, 1982; Coster and Chermant, 1985; 1989) as, according to the previous definition, $\mathrm{T}(\mathrm{K})$ corresponds to a measurement of the eroded of the complementary set by the compact $\mathrm{K}$.

The models we have chosen to test do not possess these properties. As these models are not infinitely divisible, the Voronoi tessellation nor the JohnsonMehl model, as defined in $\mathbf{R}^{3}$, does not reproduce a model of the same type in $\mathbf{R}^{2}$. The stereological aspect does not exist. To verify these models, a 3D simulation, which is intersected by a plane, must be undertaken.

The properties of calculability of these models are also poor. The analytical expressions of $\mathrm{T}(\mathrm{K})$, or derived expressions as $\mathrm{P}(\ell)$ function (in this case $\mathrm{T}(\mathrm{K})=1-\mathrm{P}(\ell)$ ), are generally not known. Let us recall 
that the $\mathrm{P}(\ell)$ function defines the probability that a segment of length $\ell$ is included in a set (Haas et al., 1967). 1- $\mathrm{P}(\ell)$ is equivalent to the linear first contact spatial distribution, $\mathrm{H}(\ell)$, while the granulometric density $\mathrm{f} \%(\ell)$ is the chord length probability density. Thus it is necessary to choose other measurements, as grain size distribution in 2D or distribution of neighbours in $2 \mathrm{D}$, with which to compare the result of intersections of 3D simulations by plane sections.

\section{VORONOI TESSELLATION}

Although the Voronoi tessellation hypotheses can often be rejected (Stoyan et al., 1995), we shall nevertheless discuss the Voronoi model for the reader not familiar with it, and who wants to test it for a given material.

\section{DEFINITION}

Consider a Poisson point process, $\mathrm{P}=\bigcup\left\{\mathrm{x}_{\mathrm{i}}\right\}$. A zone of influence is associated with each point of the process. This corresponds to the set of points y closer than any other to the point $\mathrm{x}_{\mathrm{i}}$ :

$$
X_{i}=\left\{y: d_{x_{i} y}<d_{x_{j} y}\right\} .
$$

The Voronoi model has been discussed in many publications and particularly in the book of Okabe et al. (2000) and in the revue paper by Stoyan (1998), which illustrates well its construction. The properties of the model can also be found in the book of Stoyan et al. (1995, chapter 10). In $\mathbf{R}^{3}$, tessellation can be also described as a set of convex polyhedrae. Starting from the Poisson point process, each point is linked to its nearest neighbours by a straight segment. Each segment enables the median plane to be constructed. The intersection of these planes defines the faces of polyhedrae.

\section{CONSTRUCTION}

The algorithm used to construct the Voronoi model is an algorithm of "priority growth" similar to the process used to define the watershed line (Serra, 1982; Beucher and Meyer, 1992). It can also be found on Internet. So it will not be described in our paper.

Thus a mosaic in which the boundaries correspond to a change in colour is obtained. Fig. 1 shows a 3D simulation, Fig. 2 its intersection by a plane and Fig. 3 a cross section of $\mathrm{CeO}_{2}$.

\section{CHOICE OF POINT DENSITY}

The Voronoi model depends only on one parameter: the Poisson point density, $\theta$. We then have to estimate $\theta$ from measurements on the real segmented structure. As the model is constructed in $\mathbf{R}^{\mathbf{3}}$, the stereological relationship established for the Voronoi model can be used: it provides the parameter $\theta=\mathrm{N}_{\mathrm{V}}$ (i.e., here the number of cells per unit volume, $\mathrm{N}_{\mathrm{V}}$ ) as a function of the connectivity number $\mathrm{N}_{\mathrm{A}}$ in $\mathbf{R}^{\mathbf{2}}$ (the number of polygons per unit area), (Serra, 1982):

$$
\mathrm{N}_{\mathrm{A}}(\mathrm{X})=1.46 \mathrm{~N}_{\mathrm{V}}^{2 / 3}(\mathrm{X})=1.46 \theta^{2 / 3} \text {. }
$$

Several boxes of $612 \times 468 \times 468$ pixel size were created, with 678 nuclei per box (i.e., $\mathrm{N}_{\mathrm{V}}=5.0510^{-6}$ grain/pixel ${ }^{3}$ for a specimen sintered at $1400^{\circ} \mathrm{C}$ for $1 \mathrm{~h}$ ). Various sections regularly spaced (50 pixels) have been made on these boxes to obtain images of $612 \times 468$ size, and with $512 \times 368$ format after elimination of the borders to avoid a bias in the simulation. The image of a 2D section of a simulation of a specimen sintered at $1400^{\circ} \mathrm{C}$ for $1 \mathrm{~h}$ is then compared with a SEM micrograph of the real $\mathrm{CeO}_{2}$ microstructure.

\section{TEST OF MODEL}

Stoyan et al. (1995) have proposed methods to test Voronoi tessellation. In this work, the $\mathrm{P}(\ell)$ function is calculated using a $2 \mathrm{D}$ section of a simulation and compared to its value on a segmented image, obtained from a SEM micrograph.

Fig. 4 shows that there is good agreement between the simulation and the actual microstructure. But, only one test is not sufficient to validate the model.

When 2D grain size distribution by individual analysis is evaluated, significant deviations are observed for the smallest values of the diameter D (Fig. 5).

The density of granulometry determined on plane sections of the 3D tessellation show the same characteristics as that observed in the literature (Hougardy and Stienen, 1987).

Plane sections show more small sized grains than there are in the plane sections of simulation. Hence, this model is not appropriate. This shows that to verify the validity of a model, it is necessary to test several functions. 


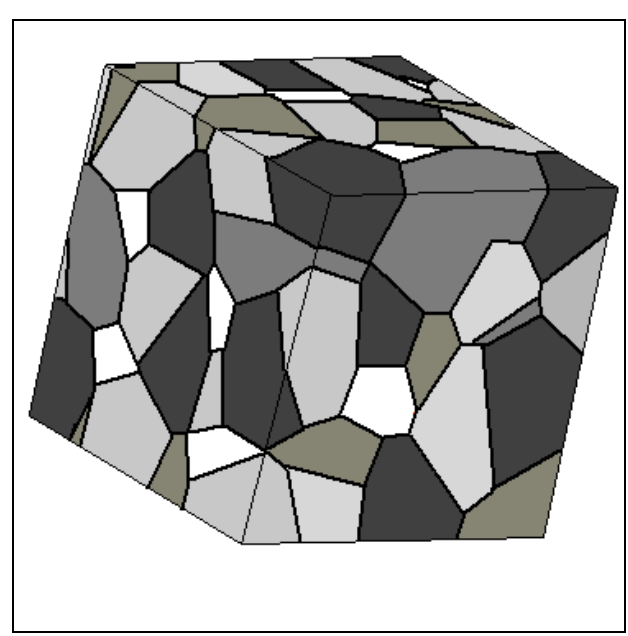

Fig. 1. 3D simulation of a Voronoi tessellation.

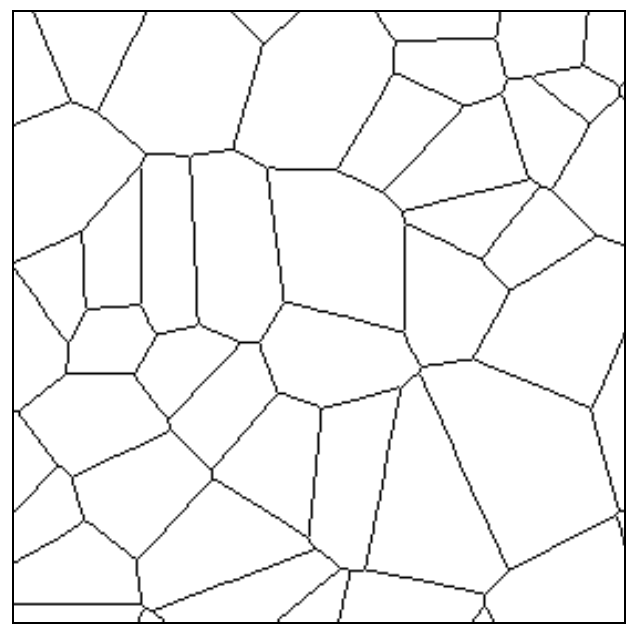

Fig. 2. Intersection by a plane of the $3 D$ simulation of Fig. 1.

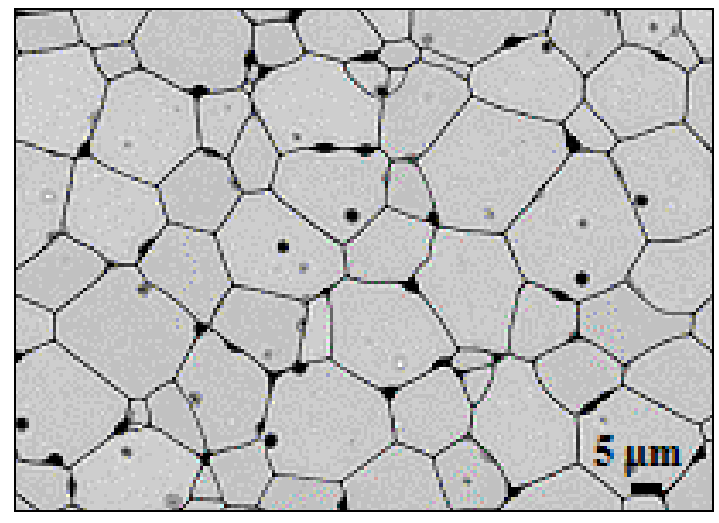

Fig. 3. Cross section of real sintered $\mathrm{CeO}_{2}$.



Fig. 4. Comparison of the $\mathrm{P}(\ell)$ function for a real $\mathrm{CeO}_{2}$ microstructure and $2 D$ sections on the Voronoi model.

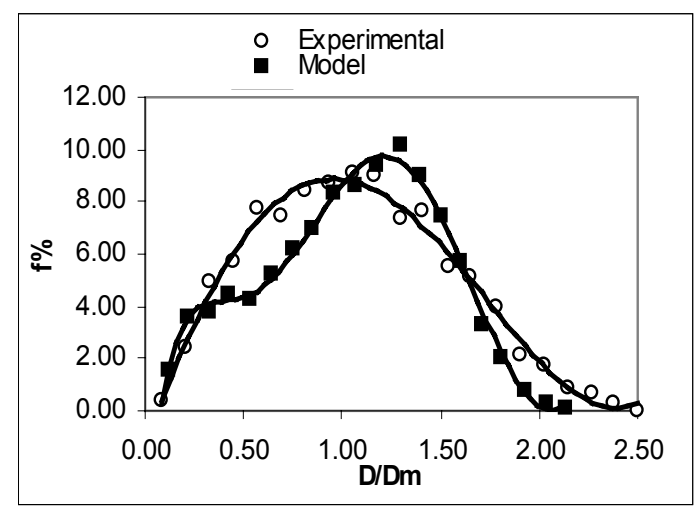

Fig. 5. Comparison of the $2 D$ granulometric density of the real $\mathrm{CeO}_{2}$ microstructure $\left(1400^{\circ} \mathrm{C}, 1 \mathrm{~h}\right)$ and $2 D$ sections on the Voronoi model.

\section{THE JOHNSON-MEHL MODEL}

\section{DEFINITION}

The Johnson-Mehl model was proposed in 1939 (Johnson and Mehl, 1939) to describe the crystalline growth of metals in the liquid phase. In order to set up a Johnson-Mehl tessellation one starts from the Poisson point process.

Contrary to the Voronoi tessellation it is a sequential model (i.e., function of time), for which there is a rate of nucleation (corresponding to an added rate of points) and also of growth, both of which can remain constant or vary with time during tessellation.

Unfortunately, this model does not possess the property of infinite divisibility. Moreover, computation is difficult and in any case, more so than for the Voronoi model. In addition, one must realize 3D simulations and undertake plane sections to measure the microstructural characteristics and to compare them to that for the real microstructure of the material. 


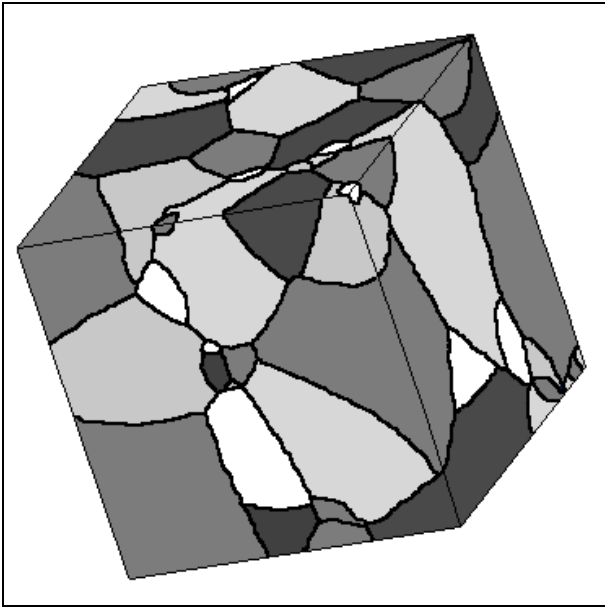

Fig. 6. 3D simulation of the Johnson-Mehl model.



Fig. 7. Intersection by a plane of the $3 D$ simulation of Fig. 6.

\section{SIMULATION OF THAT MODEL}

The algorithm is a series of nucleation stage, $\Delta \mathrm{t}_{\mathrm{n}}$, and of growth, $\Delta \mathrm{t}_{\mathrm{g}}$. During the nucleation stage, $\mathrm{n}$ nuclei are set according to a Poisson process. The rate of nucleation, $V_{n}$, is given by $n / \Delta t_{n}$. The procedure used for the growth stage $\left(\Delta \mathrm{t}_{\mathrm{g}}=1\right)$ is close to that adopted in the Voronoi model. The increase in grain size is limited to $\Delta \mathrm{D}$ for regions where growth is permitted (no other grains in the region). Growth, in fact, stops when there is contact with another grain. The growth rate, $\mathrm{V}_{\mathrm{g}}$, is defined as:

$$
\mathrm{V}_{\mathrm{g}}=\Delta \mathrm{D} / \Delta \mathrm{t}_{\mathrm{g}}=\Delta \mathrm{D}
$$

Since nuclei are created at different times the grain boundaries are not straight (in 2D) but hyperbolae contrary to the Voronoi model. Fig. 6 shows such a $3 \mathrm{D}$ simulation and Fig. 7 the result of a $2 \mathrm{D}$ section.

\section{ADJUSTMENT OF THE MODEL \\ Choice of a control parameter and a scale factor}

As shown previously, the Johnson-Mehl model depends on the rate of nucleation as given by the density of the point process $\left(\mathrm{V}_{\mathrm{n}}=\theta\right)$ and the rate of previously formed nuclei and grain growth, $\mathrm{V}_{\mathrm{g}}$.

In the Voronoi model, it is necessary to have a quantifiable parameter, which can be related to a model parameter. Since tessellation is taking place, the most relevant control parameter is the specific connectivity number, $\mathrm{N}_{\mathrm{A}}$, i.e., number of cells per unit area. In the Johnson-Mehl model, Serra (1982) has shown that this parameter is related to the number of grains per unit volume, $\mathrm{N}_{\mathrm{V}}$, as described by the relationship (in the case where $\mathrm{V}_{\mathrm{n}}$ and $\mathrm{V}_{\mathrm{g}}$ are constant rates):

$$
\mathrm{N}_{\mathrm{V}}=\left(\frac{\mathrm{N}_{\mathrm{A}}}{1.22}\right)^{\frac{3}{2}} .
$$

All size distributions measured on dense cerine show that they could be deduced from the others by a scaling factor. In other words, to complete the model and choose adjustment parameters does not necessitate a comparison with all specimens, but with one only. It is sufficient to change the pixel value for the simulated images in order to change from one real structure to another.

The simulations were carried out on boxes of size $306 \times 234 \times 234$ enabling a box of the size $256 \times 184 \times 184$ to be selected after elimination of borders. We have chosen to use this box size to reduce the time to set up the model and to carry out sectioning by two perpendicular planes to obtain 2D images with at least 95 grains $\left(\mathrm{N}_{\mathrm{A}}=2.010^{-9}\right.$ grain/ pixel $^{2}$ ). Thus, more than 3000 grains were analysed per simulation. In 3D, this corresponds to ca. 1150 nuclei $\left(\mathrm{N}_{\mathrm{V}}=6.710^{-5}\right.$ grains $/$ pixel $\left.^{3}\right)$. On reaching this number of nuclei, grain growth stops without the addition of new nuclei.

\section{Choice of the laws of nucleation and growth}

Simulations with constant nucleation and growth rates are unsatisfactory. The choice of the nucleation and growth laws is made by comparing the $2 \mathrm{D}$ size distribution of the simulation with that of the real microstructure, which corresponds to a normal law. 
In order to obtain the granulometric density, which fits a normal law, a constant growth rate $\mathrm{V}_{\mathrm{g}}$ is chosen and the nucleation rate, $\mathrm{V}_{\mathrm{n}}$ has to vary with time (iteration) according to the semi-empirical law:

$$
\mathrm{V}_{\mathrm{n}}=\mathrm{n}_{0} \exp \left[-\alpha\left(\mathrm{t}-\mathrm{t}_{\max }\right)^{2}\right],
$$

with $t_{\max }$, the iteration for which the nucleation is maximum.

Such systematical simulations enable the following granulometric behaviour to be deduced:

- for a constant growth rate, $\mathrm{V}_{\mathrm{g}}$, the higher the value of $\alpha$ is, the lower is the reduced standard deviation (RSD) of the size distribution.

- with a nucleation law where the parameters $t_{\max }$, $\mathrm{n}_{0}$, and $\alpha$ are set once and for all, the more the growth rate increases, the greater is the reduced standard deviation, which characterises the normal law.

The latter behaviour is the easiest to use if the growth rate is sufficiently high not to be undergoing a Voronoi tessellation.

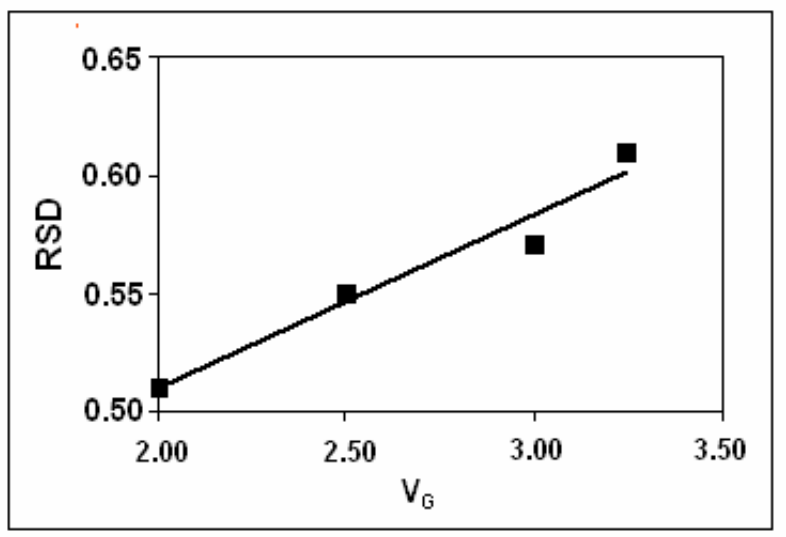

Fig. 8. Change in the reduced standard deviation, $R S D$, of the granulometric density of the simulated microstructures as function of the growth rate, $V_{g}$.

The previous studies have enabled the following nucleation expression to be established:

$$
\mathrm{V}_{\mathrm{n}}=300 \exp \left[-0.2(\mathrm{t}-6)^{2}\right] \text {. }
$$

Fig. 8 can then be used as a calibration curve, which provides the reduced standard deviation as a function of $\mathrm{V}_{\mathrm{g}}$ for the nucleation law.

\section{COMPARISON BETWEEN MEASUREMENTS ON THE MODEL AND REAL MICROSTRUCTURES}

Since the reduced standard deviation of highly densified cerine is between 0.5 and 0.6 , simulation of such microstructures is possible. As some of the specimens were fabricated at different temperatures and times, the granulometric density characterized by the same reduced standard deviation, a sole simulation is sufficient to modelise the microstructure (only the pixel value changes).

After calibration, granulometric densities can be compared of simulated and real microstructures. Fig. 9 , for example, shows the images and the granulometric densities for the microstructure of cerine (fabricated at $1450^{\circ} \mathrm{C}$ for $5 \mathrm{~h}$ ) together with the microstructure simulated from the previous nucleation rate and a growth rate $\mathrm{V}_{\mathrm{g}}$, equal to 3 .

The 2D granulometric distribution having been used to fit the model parameters, other parameters and functions must be used to replace the Choquet capacity. The following functions have been compared for the same specimen:

- the $\mathrm{P}(\ell)$ function and corresponding linear granulometries (Figs. $10 \mathrm{a}$ and $\mathrm{b}$ ),

- the distribution in number of neighbours of a grain on 2D sections (Fig. 11).

The linear granulometric distribution for the simulated and real microstructures are in good agreement. The distribution of the number of neighbours for the two images are similar to a semi-log plot but have slight differences in the standard deviation (Fig. 11). In fact, the simulated microstructures contain slightly more grains with a number of neighbours smaller than 4.

To complete the investigation, all other measurements, which enable the morphology of real microstructures to be completely described, can be validated on simulated microstructures. Results have shown that real and simulated microstructures exhibit the same morphological properties.

Table 2 shows all simulated parameters on a dense microstructure of cerine: mean diameter, specific connectivity number, intercept number, measured both on real and simulated microstructures (numbers in italics). 
Thus the use of the modified Johnson-Mehl model with a nucleation rate according to the law:

$$
\mathrm{V}_{\mathrm{n}}=300 \exp \left[-0.2(\mathrm{t}-6)^{2}\right]
$$

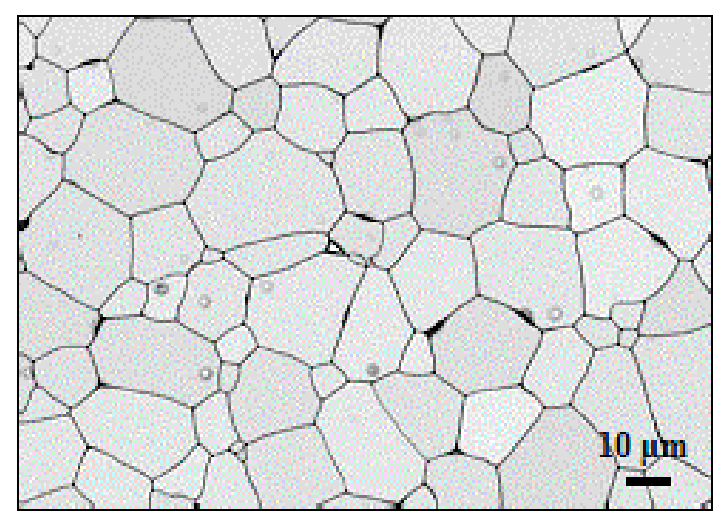

a)

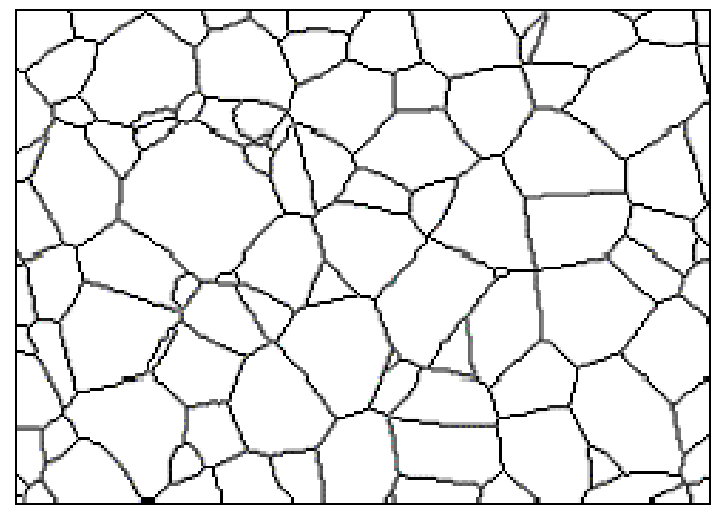

b)

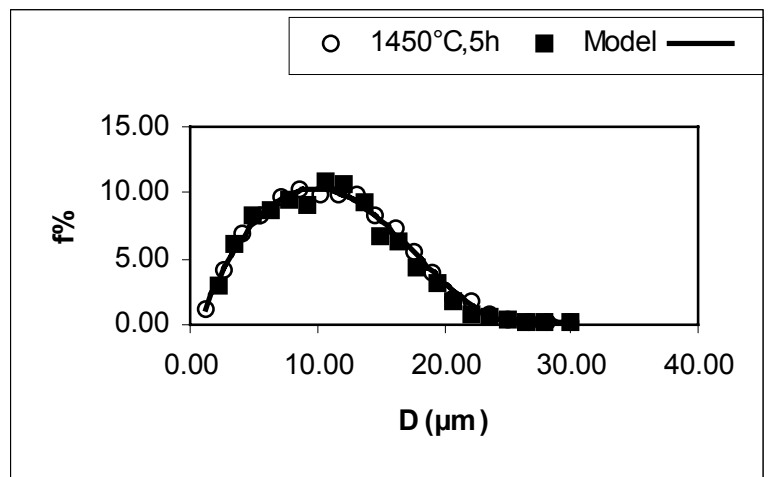

c)

Fig. 9. a) Real metallographic section of $\mathrm{CeO}_{2}$ sintered at $1450^{\circ} \mathrm{C}$ for $5 \mathrm{~h}$; b) Corresponding JohnsonMehl simulation; c) $2 D$ granulometric density for the real and simulated images of Fig. $9 a$ and $9 b$, with $V_{g}$ $=3$ and

$$
\mathrm{V}_{\mathrm{n}}=300 \exp \left[-0.2(\mathrm{t}-6)^{2}\right]
$$

and a constant rate of growth has enabled a set of dense $\mathrm{CeO}_{2}$ microstructures, despite slight variation in the reduced standard deviation of the granulometric densities to be modelled.

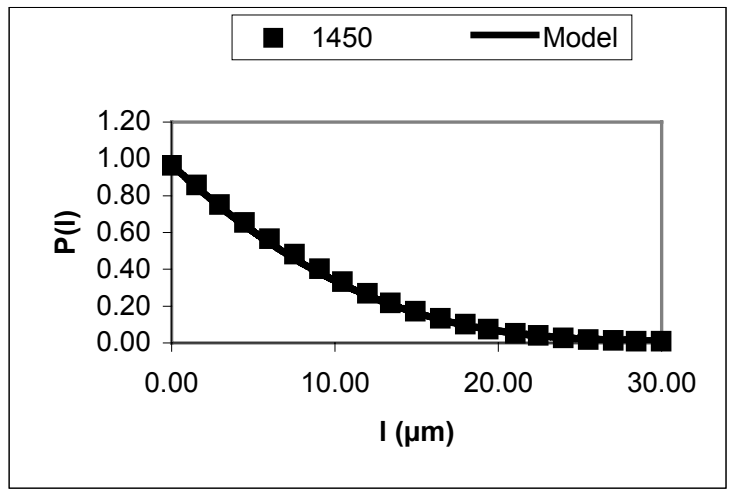

a)

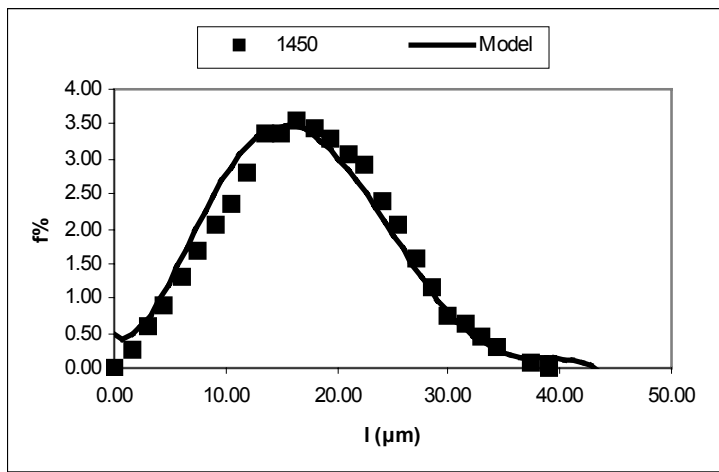

b)

Fig. 10. a) Function $P(\ell)$, and b) Linear size distribution density, $f \%$, for simulated and real $\mathrm{CeO}_{2}$ microstructure $\left(1450^{\circ} \mathrm{C}, 5 \mathrm{~h}\right)$.



Fig. 11. Distribution of the number of neighbours, $\mathrm{Nb}$, on a $2 \mathrm{D}$ section for $\mathrm{CeO}_{2}$ microstructure $\left(1450^{\circ} \mathrm{C}\right.$, 5 h) and its simulation. 
Table 2. Values of some morphological parameters measured on real and simulated cerine microstructures: First column gives the fabrication conditions; $V_{g}$ : growth rate; $D_{m}$ : the mean diameter of the cerine grains; $N_{A}$ : the connectivity number; $N_{L}$ : the intercept number. Values in italics are those for simulated microstructures.

\begin{tabular}{l|c|c|c|c}
\hline & $\mathrm{V}_{\mathrm{g}}$ & $\mathbf{D}_{\mathrm{m}}(\mu \mathrm{m})$ & $\mathrm{N}_{\mathrm{A}}\left(\mathrm{mm}^{-2}\right)$ & $\mathrm{N}_{\mathrm{L}}\left(\mathrm{mm}^{-1}\right)$ \\
\hline $1300^{\circ} \mathrm{C}, 2 \mathrm{~h}$ & 2.5 & $3.3 / 3.4$ & $73805 / 75160$ & $316 / 315$ \\
$1300^{\circ} \mathrm{C}, 5 \mathrm{~h}$ & 3 & $5.4 / 5,3$ & $29196 / 29611$ & $193 / 198$ \\
$1300^{\circ} \mathrm{C}, 10 \mathrm{~h}$ & 3.25 & $7.1 / 7.2$ & $15095 / 15879$ & $145 / 144$ \\
$1300^{\circ} \mathrm{C}, 20 \mathrm{~h}$ & 3.25 & $10.5 / 10.8$ & $6467 / 6803$ & $97 / 94$ \\
$1400^{\circ} \mathrm{C}, 18 \mathrm{mn}$ & 2.5 & $2.2 / 2.2$ & $170903 / 174048$ & $417 / 480$ \\
$1400^{\circ} \mathrm{C}, 36 \mathrm{mn}$ & 3 & $3.8 / 3.8$ & $58134 / 58747$ & $242 / 279$ \\
$1400^{\circ} \mathrm{C}, 1 \mathrm{~h}$ & 2.5 & $5.4 / 5.2$ & $30481 / 31153$ & $194 / 204$ \\
$1400^{\circ} \mathrm{C}, 2 \mathrm{~h}$ & 2.5 & $7.7 / 7.6$ & $14414 / 14690$ & $135 / 140$ \\
$1400^{\circ} \mathrm{C}, 5 \mathrm{~h}$ & 3 & $10.4 / 10.2$ & $7921 / 8007$ & $100 / 103$ \\
$1450^{\circ} \mathrm{C}, 18 \mathrm{mn}$ & 2.5 & $3.7 / 3.7$ & $60599 / 61533$ & $247 / 286$ \\
$1450^{\circ} \mathrm{C}, 36 \mathrm{mn}$ & 2.5 & $5.6 / 5.5$ & $27846 / 28255$ & $168 / 194$ \\
$1450^{\circ} \mathrm{C}, 1 \mathrm{~h}$ & 2.5 & $7.5 / 7.4$ & $15436 / 15636$ & $138 / 144$ \\
$1450^{\circ} \mathrm{C}, 2 \mathrm{~h}$ & 3 & $9.3 / 9.2$ & $9811 / 9886$ & $111 / 114$ \\
$1450^{\circ} \mathrm{C}, 5 \mathrm{~h}$ & 3 & $14.0 / 13.9$ & $4259 / 4303$ & $74 / 75$ \\
\hline
\end{tabular}

\section{TOWARDS A PROBABILISTIC MODEL FOR POROUS MATERIALS}

The previous model can serve as a basis to describe sintered materials when the pores are closed. For this "reversible virtual sintering" is proposed which retraces the various sintering stages from the dense structure to the creation of pores.

The growth of pores and then their progressive elimination are generally the final sintering stages. The reverse phenomenon consists of pore creation in a granular structure. The hypothesis is that pores preferentially form at triple points (boundaries in 3D). Of course on real materials, these pores are very rarely spherical! If granular structure is labelled, these triple points can easily be located on a simulation as the corresponding voxels are neighbours with different labels.

The number and size of pores to be randomly implanted on these triple boundaries are estimated from the stereological parameters measured on real microstructures. Obviously, there is a stereological relationship (DeHoff, 1968) between the number of pores (supposed to be convex) per unit volume, $\mathrm{N}_{\mathrm{V}}(\mathrm{P})$, their number per unit area, $\mathrm{N}_{\mathrm{A}}(\mathrm{P})$, and their mean Ferret diameter, $\mathrm{D}_{\mathrm{Fm}}$ :

$$
\mathrm{N}_{\mathrm{A}}(\mathrm{P})=\mathrm{D}_{\mathrm{Fm}} \mathrm{N}_{\mathrm{V}}(\mathrm{P}),
$$

$\mathrm{N}_{\mathrm{A}}(\mathrm{P})$ is estimated from the microstructure, but $\mathrm{D}_{\mathrm{Fm}}$ is not known as it would be necessary to have the 3D granulometric distribution. Hence the porous model has been modified, supposing a single size of sphere. In order to establish the pertinence of this assumption, the covariance function of a porous network for various sintering times at $1100^{\circ} \mathrm{C}$ was compared to that of the simulation (Fig. 12). Indeed, covariance function is the tool the best adapted to analyse the dispersion of a phase in the other one.

It should be noted that this covariance function passes through a minimum for all investigated $\mathrm{CeO}_{2}$ materials. This demonstrates, that there is a geometrical effect of pore repulsion. Consequently, two very close pores coalesce very quickly to form only one pore. In a simulation with a random implant of pores, the covariance function can only decrease monotonically. We therefore introduced a repulsive force in the mode, which imposed a minimum distance between two neighbouring pores. Fig. 13 shows the obtained results obtained under the following conditions:

- $\mathrm{V}_{\mathrm{g}}=2$,

- $\quad V_{n}=300 \exp \left[-0.2(t-6)^{2}\right]$,

- number of pores in the 3D box $=2000$,

- pore diameter $=10$ voxels,

- repulsion distance $=2$ voxels. 


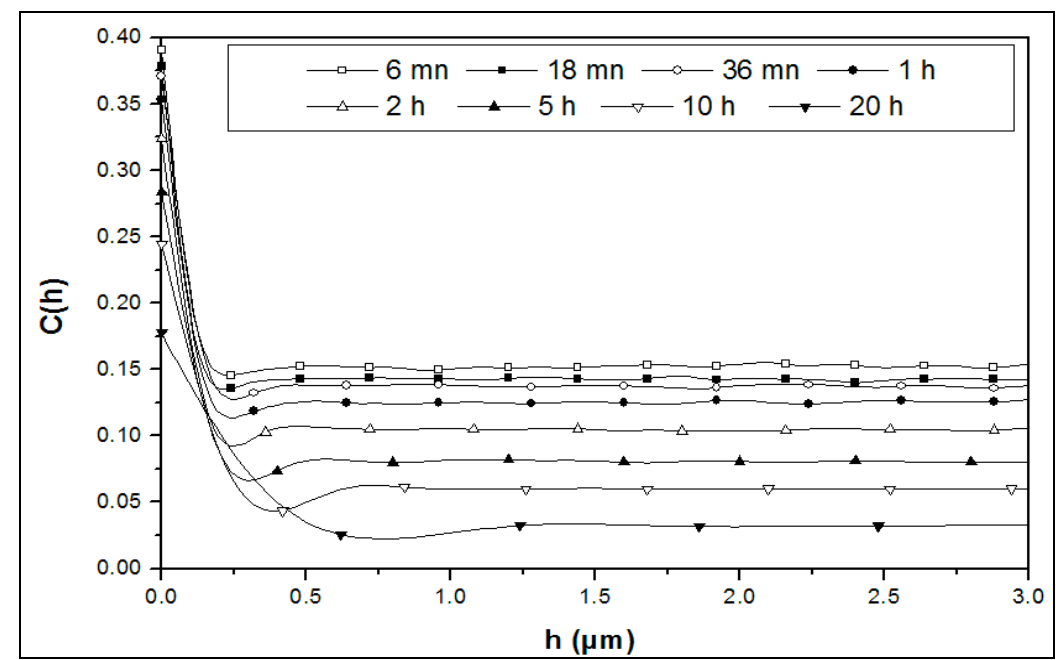

Fig. 12. Covariance function, $\mathrm{C}(\mathrm{h})$, of the porous network for various times of sintering of $\mathrm{CeO}_{2}$ at $1100^{\circ} \mathrm{C}$.

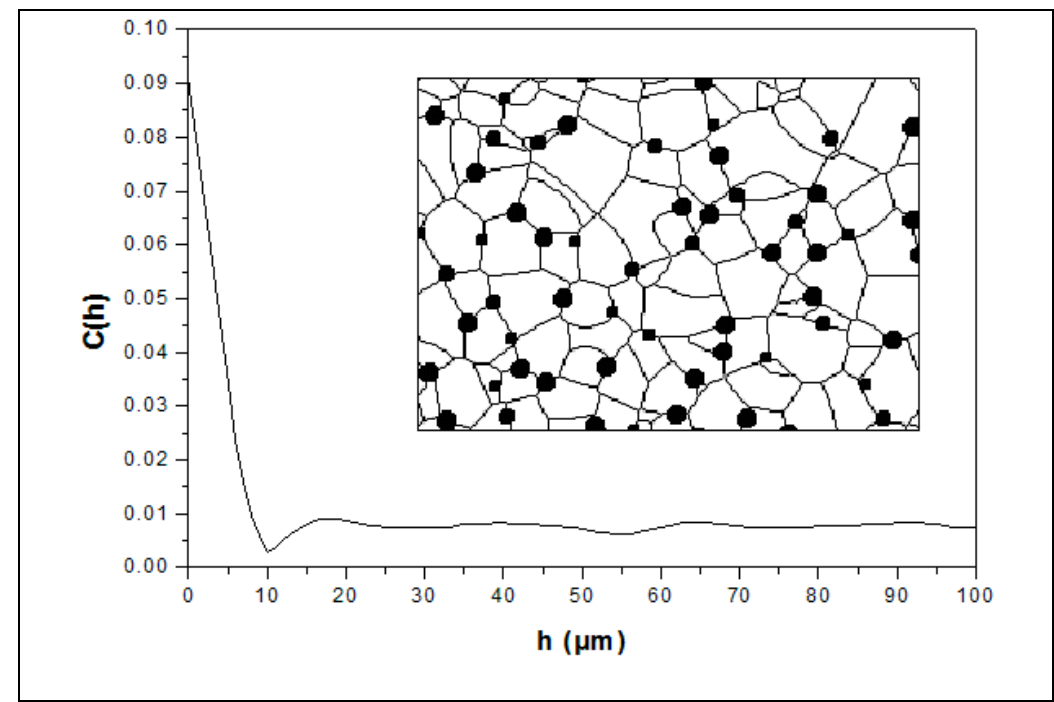

Fig. 13. Covariance function, $C(h)$, of the simulated microstructure and representation of a $2 D$ section of the porous material.

These first results are encouraging enough to consider an extension of the model so long as there are still pores. The model could be improved, in particular, with respect to the comparison of the $2 \mathrm{D}$ distribution of the pores in a real material and in fitting a $3 \mathrm{D}$ distribution, taking into account the granulometric parameters and the shape of the grains. In our approach we have only used monosized spheres located at the edges of the 3D tessellation.

This demonstrates the flexibity of the approach used in combining the simple probabilistic models and coupling this construction to a comparison by microstructural analysis of the model and the real structure by mathematical morphology. This was the approach adopted by Dequiedt et al. (2000). Following the same concept, it is possible to implant defects randomly in a model of a perfect microstructure.

\section{CONCLUSION}

In this work two probabilistic models were used to simulate the morphology of a monophased granular ceramic: cerine, $\mathrm{CeO}_{2}$ : the Voronoi tessellation and the Johnson-Mehl model.

The Voronoi tessellation is not convenient (as even already shown by Stoyan et al., 1995), as the simulated granulometric densities are different from those of the real material: they do not fit a log-normal law. 
The best results were obtained with the JohnsonMehl model as it integrates a sequential nucleation and encompasses more parameters. All sets of dense $\mathrm{CeO}_{2}$ sintered specimens were modelled based on a nucleation rate obeying a law derived from "Gaussian pattern", and a constant growth rate.

Very good agreement was observed for the granulometric characteristics of the sectioning of 3D simulations and also those of real ceramographic sections. An extension of the model is also proposed for porous microstructures.

Compared to "granular models", as described by Horalèk (1988; 1990), and to results obtained from other types of models already published by many authors (for example computer-simulated grain structure, discrete numerical modelling and packing of spheres, models based on finite element methods, etc.) as listed in the introduction, this work shows that probabilistic models are admirably suited for the simulation of ceramic and granular microstructures in addition to concrete (Dequiedt et al., 2000), and require only a few parameters to describe the morphology. Moreover, many of them possess stereological properties: this implies that you can measure morphological parameters on a 2D section and, for example, obtain the number of objects per unit volume without any hypothesis. Let us recall that this parameter can only be measured experimentally by serial sectioning unless you make an hypothesis. Experimentally, it is very difficult to carry out correctly and it is very time consuming.

\section{ACKNOWLEDGEMENTS}

This work was performed at LERMAT (URA CNRS 1317, ISMRA, Caen, France) in the frame of the CRITIC (Comité Régional de l'Image et des Technologies de l'Information et de la Communication) Pole, that we thank. It has been financially supported by two contracts: "Granulométrie et modé-lisation de céramiques", $1999 \mathrm{n}^{\circ}$ 99.12, and "Modélisation et logiciel de granulométrie de matériaux granulaires", $2001 \mathrm{n}^{\circ} 01.285$. The authors, and more specially X. Arnould, are grateful to ADCIS, Caen, France, for its financial support and discussions.

Many thanks, to one of the referees for his constructive comments and to Prof. B.L. Mordike from the University of Malta for fruitful discussions.

\section{REFERENCES}

Arnould X (2002). Granulométrie et modélisation de céramiques - granulometry and modelling ceramics. Thèse de Doctorat of the University of Caen.
Arnould X, Coster M, Chermant JL, Chermant L, Chartier T, El Moataz A (2001). Segmentation and grain size of ceramics. Image Anal Stereol 20(suppl. 1):131-5.

Arnould X, Coster M, Chermant JL, Chermant L, Chartier $\mathrm{T}$ (2002). Ceramography and segmentation of polycristalline ceramics: application to grain size analysis by automatic methods. $7^{\text {th }}$ Congress of the European Ceramic Society. 7 ECRS, Brugge, Belgium, Sept. 9-13, 2001. Trans Tech Pub, Key Eng Mater 206:685-90.

Aubert A, Jeulin D (2000). Classification morphologique de surfaces rugueuses. Rev Mét CIT Sci Génie Mater 97(12):247-62.

Bentz DP (1997). Three-dimensional computer simulation of Portland cement hydration and microstructure development. J Amer Ceram Soc 80:3-21.

Beucher S, Meyer F (1992). The morphological approach of segmentation: the watershed trans-formation. In: Dougherty EM, ed. Mathematical Morphology in Image Processing. New York: Dekker, 433-81.

Choquet G (1953). Theory of capacities. Ann Inst Fourier 5:131-295.

Coster M, Chermant JL (1985). Précis d'Analyse d'Images $1^{\text {st }}$ ed. Paris: Presses du CNRS.

Coster M, Chermant JL (1989). Précis d'Analyse d'Images. $2^{\text {th }}$ ed. Paris: Les Editions du CNRS.

Coster M, Chermant JL (2002). On a way to material models for ceramics. J Eur Ceram Soc 22:1191-203.

Coster M, Arnould X, Chermant JL, Chermant L, Chartier $T$ (2005). The use of image analysis for sintering investigations. The example of $\mathrm{CeO}_{2}$ doped with $\mathrm{TiO}_{2}$. J Eur Ceram Soc 25. (2005 in press)

DeHoff RT (1968). Quantitative Microscopy. New York: Mc GrawHill.

Dequiedt AS, Coster M, Chermant JL, Jeulin D (2000). Towards a model of concrete microstructure. Cem Concr Comp 22:289-97.

Dinger DR (2000). One-dimensional packing of spheres, Part II. Amer Ceram Soc Bull 79:83-91.

Garboczi EJ, Bentz DP (1993). Computational materials science of cement based materials. Mat Res Bull 18: 50-4.

Ghosh S, Nowak Z, Kyunghoon L (1997a). Tessellationbased computational methods for the characterization and analysis of heterogeneous microstructures. Comp Sci Tech 57:1187-210.

Ghosh S, Nowak Z, Kyunghoon L (1997b). Quantitative characterization and modelling of composites microstructures by Voronoi cells. Acta Mater 45-6:2215-34.

Haas A, Matheron G, Serra J (1967). Morphologie mathématique et granulometries en place. Ann Mines $11: 736-53$ and 12:767-82.

Horalèk V (1988). A note on the time non-homogeneous Johnson-Mehl tessellation. Adv Appl Prob 20:684-5. 
Horalèk V (1990). ASTM grain size model and related random tessellation models. Materials Characterization. 25:263-84.

Hougardy H, Stienen H (1987). Computer simulation of carbide growth in steel during annealing. Acta Stereol $6(1): 175-80$.

Jeulin D (1991). Modèles Morphologiques de Structures Aléatoires et de Changement d'Echelle. Thèse de Doctorat dès Sciences. University of Caen.

Jeulin D (1997a). Advances in theory and applications of random sets. Singapore: World Scientific.

Jeulin D (1997b). Dead leaves models, from space tessellations to random functions. In: Jeulin D, ed. Advances in theory and applications of random sets. Singapore: World Scientific, 137-56.

Jeulin D, Laurenge P (1996). Morpho-logical simulation of the roughness transfer on steel sheets. Microsc Microanal Microstruct 7:541-7.

Johnson WA, Mehl RF (1939). Reaction kinetics in processes of nucleation and growth. Trans AIME 135:416-58.

Matheron G (1975). Random Sets and Integral Geometry. New York: John Wiley \& Sons.

Matsubara H, Nomura H, Kitaoka S (1999). Design of ceramic microstructures by Monte Carlo simulations. Key Eng Mater 161-163:33-8.

Mehnert K, Ohser J, Klimaneck P (1998). Testing stereological methods for estimation of spatial size distributions by means of computer-simulated grain structures. Mater Sci Eng A256:207-12.

Miles RE (1971). Poisson flats in euclidean spaces. Part 2: Homogeneous Poisson flats and the complementary theorem. Adv Appl Prob 3:1-43.
Okabe A, Boots B, Sugihara K, Chiu SN (2000). Spatial tessellations: Concepts and applications of Voronoi diagrams (POD). New York: John Wiley \& Sons.

Serra J (1982). Image analysis and mathematical morphology. London: Academic Press.

Serra J (1988). Image analysis and mathematical morphology, Vol. 2, Theoretical Advances. London: Academic Press.

Stoyan D (1998). Random sets, models and statistics. Int Stat Rev 66:1-27.

Stoyan D, Kendall WS, Mecke J (1995). Stochastic geometry and its applications, $2^{\text {nd }}$ edition. New York: John Wiley \& Sons.

Stroeven M (1999). Discrete numerical modelling of composite materials. Application to cementious materials. PhD Thesis, Technische Universität Delft.

Stroeven M, Stroeven P (1996). Computer-simulated internal structure of materials. Acta Stereol 15(3): 24752 .

Tikare V, Cawley JD (1998a). Numerical simulation of grain growth in liquid phase sintered materials - I. Model. Acta Mater 46-4:1333-42.

Tikare V, Cawley JD (1998b). Numerical simulation of grain growth in liquid phase sintered materials - II. Study of the isotropic grain growth. Acta Mater 46-4: 1343-56.

Xi Y, Tennis PD, Jennings HM (1996). Mathematical modelling of cement paste microstructure by mosaic pattern. Part I. Formulation. J Mater Res 11:1943-52.

Xiaoyan S, Liu G (1998). A simple and efficient threedimensional Monte Carlo simulation of grain growth. Scripta Mater 38-11:1691-6. 